\title{
"Cahul" ozonated mineral water in the treatment of experimentally induced chronic rhinosinusitis
}

\section{Viorica Chistruga-Blajin, Sandul Alexandru}

Department of Otorhinolaryngology of the "Nicolae Testemitanu" State University of Medicine and Pharmacy, Chisinau, Republic of Moldova

\section{ABSTRACT}

BACKGROUND. Chronic maxillary sinusitis is a frequently encountered pathology, resistant to treatment, which can cause significant economic losses. Considering these aspects, it is necessary to develop new methods of treatment.

OBJECTIVE. The purpose of this study was to assess the therapeutic efficacy of the "Cahul" ozonated mineral water in the treatment of experimentally induced chronic rhinosinusitis and its action on the mucosa of the maxillary sinus.

MATERIAL AND METHODS. The experimental study was conducted on a group of 14 chinchilla rabbits, aged between 8 and 12 months, weighing 2.5-3.0 kg, kept in vivarium conditions. In the first stage of the study, chronic rhinosinusitis was induced. 3 months after, maxillary antrostomy in the medial wall was performed on all animals included in the study; the intervention was performed under general anesthesia. The rabbits were divided into two groups. The rabbits enrolled in the study group underwent daily inhalations and maxillary sinus lavage with the "Cahul" ozonated mineral water. In the control group, inhalations and lavage were performed with $0.9 \% \mathrm{NaCl}$ solution. Efficacy of the treatment was assessed observing the clinical character of the pathology. On days 2, 5 and 9 of the experiment, endoscopic and histological examinations were performed.

RESULTS. On the ninth day of treatment, if in the study group the appearance of the sinus mucosa was almost normal, in the control group, the histological examination revealed: epithelial hyperplasia, cylindrical cell hyperplasia, thickening of the basement membrane and lymphocyte infiltration. Mucosal fragments harvested after 5 days revealed submucosal fibrosis, leukocyte infiltration in the control group being more pronounced than in the study group.

CONCLUSION. Maxillary sinus lavage with the "Cahul" ozonated mineral water improves mucociliary clearance and stimulates regeneration of affected cells.

KEYWORDS: rabbit, model, chronic rhinosinusitis, mineral water, ozone.

\section{INTRODUCTION}

Inflammation of the sinus cavities involves a number of disorders, including acute and chronic maxillary rhinosinusitis. Evolution of acute maxillary rhinosinusitis is dependent on the correctness of the treatment applied and on the clinico-anatomical form of the disease; it can heal or become chronic with the occurrence of irreversible histological changes in the sinus mucosa.

Globally, in the treatment of paranasal sinuses pathology, important medical resources are used, with great economic losses. In this group of disorders, chronic maxillary rhinosinusitis is a frequent pathology resistant to treatment ${ }^{1}$.
More than $20 \%$ of the global population suffers from this pathology. In the last 10 years, diseases have increased 3 times, but the number of patients admitted with this paranasal sinus pathology increases annually by 1.5 to $2 \%^{2}$.

In the last decade, a visible progress has been observed in the diagnosis and treatment of the pathology of the respiratory system, and especially in the case of different forms of rhinosinusitis ${ }^{3}$.

Despite numerous studies existing in the literature, pathogenesis of chronic sinusitis is not well known and there is still no treatment with minimal side effects and significant clinical results ${ }^{3}$. 


\section{MATERIAL AND METHODS}

This study was conducted under the supervision of a veterinary specialist. All procedures that were performed and the living conditions of rabbits included in the study were approved and monitored by the Ethics Commission of the "Nicolae Testemitanu" State University of Medicine and Pharmacy.

The parallel experimental study was carried out in the laboratory of the Public Institution "Republican Centre of Veterinary Diagnosis", Cahul subdivision. The animals selected for the study were rabbits, because the nasal anatomical structure of these animals is similar to that of humans, thus providing optimum manipulation, monitoring and evaluation conditions.

The experimental study was carried out on a group of 14 adult chinchilla rabbits, aged between 8 and 12 months and weighing $2.5-3.0 \mathrm{~kg}$, kept in vivarium conditions.

In the first stage of the study, chronic rhinogenic maxillary rhinosinusitis was induced after the model patented and published by Liang in $2008^{4}$. We also took into consideration the comparative study of several modelling techniques of experimental sinusitis in rabbit, conducted by Henrique Olival Costa and the co-authors ${ }^{5}$. The pattern of rhinosinusitis induced with a sponge causes a more intense inflammation, it is easy to perform and reversible by treatment.

3 months after, maxillary antrostomy in the medial wall of each infected maxillary sinus, by external approach, was performed on all animals, under general anesthesia.

Before the surgical procedure, the animals were anesthetized intramuscularly with $10 \mathrm{ml}(10 \mathrm{mg} / \mathrm{kg})$ of Ketamine hydrochloride solution $5 \%$, spontaneous ventilation being maintained.
The incision was performed and the periosteum taken off (Figure 1a). The flap was elevated laterally and, with a small milling cutter of $3 \mathrm{~mm}$ in diameter, the milling of the upper wall of the affected maxillary sinus was carried out (Figure 1b). Mucosa fragments for pathological examination and samples for bacteriological examination were taken. A drain of $0.4 \mathrm{~cm}$ in diameter was mounted, fixed by suture; six sutures were applied on the flaps. The duration of each surgery varied between 15 to 20 minutes.

For treatment, the animals were divided into two groups:

- The study group - 7 rabbits - daily lavage of the maxillary sinus and inhalations with the "Cahul" ozonated mineral water.

- The control group - 7 rabbits - lavage of the sinus and inhalations with $0.9 \% \mathrm{NaCl}$ solution.

The treatment in both groups started 3 months after inoculation of the foreign body in the nasal cavity and lasted 9 days.

Efficacy of the treatment was assessed depending on the clinical evolution of the pathology. On days 2, 5 and 9 of the experiment, endoscopic examination was performed with sampling of material for the morphopathological examination.

On the first and the last days, samples were taken for the bacteriological analysis of the affected maxillary sinus content, in the result of which were detected Staphylococcus aureus, E. coli, coagulase-negative staphylococcus, Pseudomonas aeruginosa. Microbiological samples were taken from the sinus with sterile cotton swabs, subsequently introduced in test tubes with $5 \mathrm{ml}$ of sterile broth. All culture samples were immediately transported to the microbiological laboratory. In the laboratory, cultures were performed, isolation and identification of microorganisms being

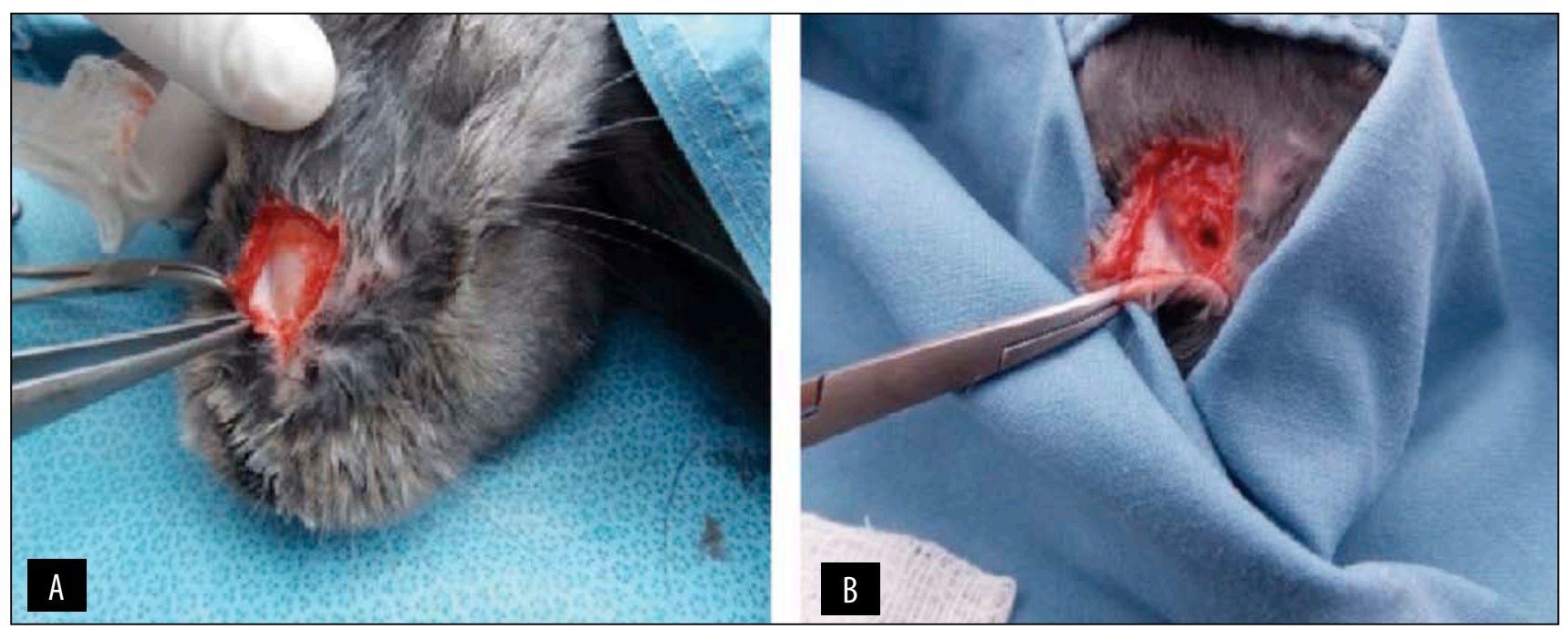

Figure 1 (A) alncision and taking off of the periosteum until visualising the bony anterior wall of the maxillary sinus. (B) antrostomy of the maxillary sinus. 
carried out according to standard microbiological procedures. Microbiological results were negative in two rabbits. 15 days after the procedure of rhinosinusitis induction, all rabbits included in the study began to show unilateral purulent rhinorrhea. After 3 months, hypertrophy of the maxillary sinus mucosa in all 14 rabbits and purulent collections in 11 of them were evident. These changes were also supported by the histological examination that revealed chronic active inflammation in the samples harvested intraoperatively.

Biopsy materials after staining with H\&E (hematoxylin eosin) were examined with the optical microscope, morphological changes proving the epithelial injury of the sinus mucosa being identified.

\section{RESULTS}

Histological analysis of the endosinusal mucosa taken during surgery showed the existence of sinus mucosal thickening, with epithelial hyperplasia, mucous metaplasia, moderate to severe subepithelial fibrosis. Moreover, one observed an obvious infiltration of leukocytes in the lamina propria and the epithelium, with predominantly mononuclear cell infiltrates (lymphocytes, macrophages and plasmocytes) and lymphoid follicular hyperplasia (Figure 2). At the time of surgery, a discrete inflammation was identified in 1 rabbit $(7.1 \%)$ (10-30 monocytes/field 40x), moderate inflammation in 4 rabbits $(28.6 \%)$, and the majority (9 rabbits, representing 64.3\%) showed an intense inflammation of grade 3 ( $>50$ monocytes/field $40 x$ ).

A day before the start of treatment, on the 2 nd and the 5 th days of treatment, the nasal cavity was examined by anterior rhinoscopy. While performing the antrostomy and on the sixth day, mucosa from the maxillary sinus was collected for histological examination.

It is well known that small animals heal faster than people ${ }^{6-8}$. On the first day of treatment, the general condition of animals was visibly affected; they were less active, with dishevelled fur. Rabbits showed symptoms characteristic of a sinus inflammatory process, manifested by: periodic sneezing, nasal obstruction, purulent rhinorrhea, hyperemia and edema of the nasal mucosa. The temperature varied no more than $0.8^{\circ} \mathrm{C}$ $\left(\mathrm{N}-38.5-39.5^{\circ} \mathrm{C}\right)$; during the study, animals did not have fever. On endoscopic examination of the maxillary sinuses, an obvious hyperemia and a mucosal edema were observed, with a pronounced vascular pattern and purulent secretions surrounding a yellowish fragmented foreign body (sponge).

\section{The study group}

On the fifth day of treatment, positive changes in the behaviour of animals have already been seen, nasal breathing being improved. Endoscopic examination revealed decreased hyperemia and edema in the endosinusal mucosa, and purulent rhinorrhea disappearance. Histological examination showed a sinus mucosa with a real tendency to restore the normal structure, with persistent signs of moderate chronic inflammation.

During treatment, the condition of animals in this group had an intensely positive dynamics.

On the ninth day of treatment, the rabbits in the study group had a satisfactory condition, with nasal breathing within normal limits. Endoscopic examination showed edema and hyperemia disappearance in the sinus mucosa.

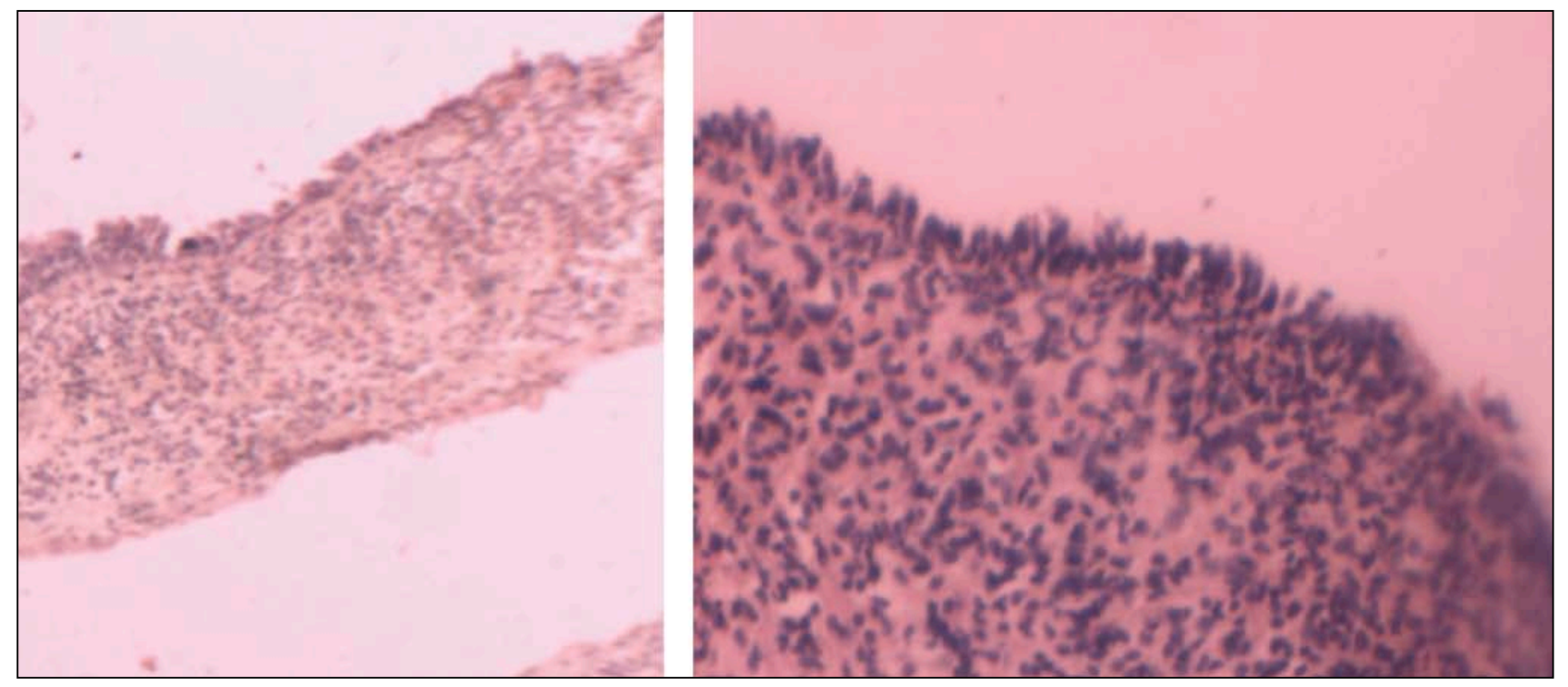

Figure 2 Histopathological examination - HE stain - thickening of the sinus mucosa, lymphoid hyperplasia, cilia degeneration, mononuclear cells infiltration, eosinophils 
Histologically, in 6 cases $(85.7 \%)$ the mucosa was represented by a multi-layered prismatic ciliated epithelium, with a smooth surface and correctly oriented polar cells, 1 rabbit $(14.3 \%)$ presented a moderate epithelial hyperplasia and discrete inflammatory signs (10-30 monocytes/field 40x).

\section{The control group}

The assessment performed on the fifth day of treatment showed no positive essential changes. The rabbits in the control group were less active, with persistent mucosal edema and purulent secretions.

The histopathological examination of the harvested material showed signs of inflammation, the stroma being edematous, with dilated capillaries and inflammatorily infiltrated in the submucosal layer. The cells of the ciliated epithelium showed signs of dystrophy, a lesion of metaplastic type in which the coating epithelium of ciliated cylindrical type is replaced in some areas with stratified squamous epithelium type.

On the ninth day of treatment, unlike the study group, rabbits in the control group still presented signs of inflammation both in the general condition, as well as on endoscopic examination. Rabbits became more active and, although purulent rhinorrhea disappearance was noted, a moderate hyperemia of the sinus mucosa persisted.

If in the study group the histological examination revealed a mucosa with normal structure in 6 rabbits $(85.7 \%)$, in the control group, the processes characteristic of chronic inflammation were still present in 5 cases $(71.5 \%)$ : epithelial hyperplasia, cylindrical cell hyperplasia, thickening of the basement membrane and lymphocyte infiltration, 1 rabbit (20\%) presented a discrete inflammation (10-30 monocytes/field 40x), 3 rabbits $(60 \%)$ with moderate inflammation $(40-50$ monocytes/field 40x) and 1 rabbit (20\%) with an intense inflammation ( $>50$ monocytes/field $40 \mathrm{x}$ ).

\section{DISCUSSIONS}

Our study performed on 14 rabbits was implemented after thorough study of the literature data. Animal experiments in the study of sinus pathologies dates back to the early twentieth century ${ }^{9,10}$. In the literature, various attempts to create animal models for this type of study are described, rodents being the most frequently encountered. There seems to be a pathophysiological reason for this, since the anatomy and physiology of the sinus complex of these animals is similar to that of human beings. The rabbit is often promoted as experimental animal of choice, due to its generous size and sinus accessibility ${ }^{11,12}$.

Kelemen ${ }^{13}$ has studied the nasal and sinus cavities in rabbits, both macroscopically and microscopically, and has described all structural details. Like in humans, the nasal cavity in the rabbit is sagittally separated by the septum, and the lateral nasal wall contains structures that are similar to the nasal turbinates in humans. The anterior structure is called maxilloturbinate, and the posterior one endoturbinate. With its broad and notched surface, the maxilloturbinate has a role in the humidification and heating of inhaled air. Although the maxilloturbinate is equivalent to the inferior nasal concha in humans, the endoturbinate can be compared to the middle nasal concha. Paranasal sinuses in humans consist of a number of cavities connected to each other. But in a rabbit model, all these cavities can be considered as a single one, called the maxillary sinus ${ }^{13}$.

Similar to the pathogenesis of human rhinosinusitis, induction of acute rhinosinusitis in rabbits requires a mechanical obstruction of the ostium. In the literature, several models of experimental sinusitis in rabbits are described ${ }^{4,8,9,12,14-17}$. The basic technique in most models is to surgically open the maxillary sinus through an incision and perform an antrostomy in the anterior wall. The natural ostium of the sinus is then sealed with a piece of cotton, either with cyanoacrylate, polyacrylate, tissue adhesive (butyl cyanoacrylate), or bone wax. After suturing the incision, standard pathogenic bacteria are injected at the level of the ostial obstruction $8,15,16,18-20$.

A group of researchers led by Henrique Olival Costa $^{5}$ conducted four techniques of experimental models in rabbits in order to obtain a sinus inflammation. The most intense inflammation was obtained by introducing a sponge in the nasal passage. An insignificant inflammatory process was observed in the model in which blood is placed in the nasal cavity.

A similar pattern is the method of obtaining chronic rhinosinusitis, described by Liang and collaborators $^{4,17}$. Due to this experimental model, we have managed to investigate a new method of treatment for chronic rhinosinusitis with "Cahul" ozonated mineral water.

Nowadays, ozone therapy represents an attractive and effective therapy, with minimal side effects. The main properties of ozone are: optimization of pro- and antioxidant systems, antibacterial, antifungal, antiviral, antiinflammatory and immunomodulatory, systemic regulation of homeostasis in the body, as well as optimization of organs and organ systems function. ${ }^{16,19,21,22}$.

The curative effect of the "Cahul" mineral water is determined by its chemical composition, the bactericidal property, the degree of improvement of local blood circulation, as well as by other factors (it acts as an anti-inflammatory, detoxicant, desensitizer, stimulator of damaged cells regeneration) ${ }^{2}$. 


\section{CONCLUSIONS}

According to the literature data and the result of our own experiments, the experimental model for inducing chronic rhinosinusitis in rabbits by using the sponge is of choice, because it causes a lasting inflammatory process, without using pathogenic agents. Maxillary sinus lavage with ozonated "Cahul" mineral water improves local blood circulation and mucociliary clearance; it stimulates regeneration of affected cells. All surgical wounds have healed without complications. Throughout the study, no data have been reported regarding the negative action or the presence of a prejudice of the ozonated "Cahul" mineral water on the maxillary sinus mucosa.

\section{Conflict of interest}

The authors have no financial or non-financial conflict of interest to declare.

\section{REFERENCES}

1. Dejima K., Randell S.H., Stutts J., Senior B.A., Boucher R.C. - Potential Role of Abnormal Ion Transport in the Pathogenesis of Chronic Sinusitis. Arch Otolaryngol Head Neck Surg., 2006;132(12):1352-1362. doi:10.1001/archotol.132.12.1352.

2. Piskunov G.Z., Piskunov S.Z. - Clinical Rhinology. Moscow: Miklon, 2002 (in Russian).

3. Ivanchenko O.A., Lopatin A.S. - Chronic rhinosinusitis: epidemiology, classification, etiology, and pathogenesis. The current view of the problem. Vest Otorinolaringol., 2012;12(2):91-96 (Article in Russian).

4. $\quad$ Liang K.L., Jiang R.S., Wang J., Shiao J.Y., Su M.C., Hsin C.H., et al. Developing a rabbit model of rhinogenic chronic rhinosinusitis. Laryngoscope, 2008;118(6):1076-1081.

5. Costa H.O., et al. - Comparative study of several sinusitis experimental modelling techniques in rabbits. Rev Bras Otorrinolaringol., 2007;73(5):627-631.

6. Sejima T., Kajiwara D., Kikuchi H., Imayoshi S., Yamauchi T., Ichimura K. Experimentally induced eosinophilic polyps in rabbit sinuses. Am J Rhinol Allergy, 2010;24(5):341-347. doi: 10.2500/ajra.2010.24.3519.
7. Bleier B.S., Kofonow J.M., Hashmi N., Chennupati S.K., Cohen N.A. Antibiotic eluting chitosan glycerophosphate implant in the setting of acute bacterial sinusitis: a rabbit model. Am J Rhinol Allergy, 2010;24(2):129-132. doi: 10.2500/ajra.2010.24.3439.

8. Kara C.O., Cetin C.B., Colakoglu N., Sengul M., Pakdemirli E. Experimentally induced rhinosinusitis in rabbits. J Otolaryngol., 2002;31(5):294-298.

9. Hilding A.C. - Experimental sinus surgery: effects of operative windows on normal sinuses. Ann Otol Rhinol Laryngol., 1941;50:379-392.

10. Knowlton C.D., McGregor G.W. - How and when the mucous membrane of the maxillary sinus regenerates: an experimental study in the dog. Arch Otolaryngol., 1928;8:647-656.

11. Gizurarson S. - Animal models for intranasal drug delivery studies:a review article. A review article. Acta Pharm Nord., 1990;2(2):105-122.

12. Illum L. - Nasal delivery. The use of animal models to predict performancein man. J Drug Target., 1996;3(6):427-442.

13. Kelemen G. - The nasal and paranasal cavities of the rabbit in experimental work. AMA Arch Otolaringol., 1955;61(5):497-512.

14. Perez A.C., Cunha S., Fialho S.L., Silva L.M., Dorgam J.V., Murushima A.A.B., Silva A.R., Rossato M., Anselmo-Lima W.T. - Assessing the maxillary sinus mucosa of rabbits in the presence of biodegradable implants. Braz J Otorhinolaryngol., 2012;78(6):40-46.

15. Johansson P., Kumlien J., Carlsoo B., Drettner B., Nord C.E. - Experimental acute sinusitis in rabbits: A bacteriological and histological study. Acta Otolaryngol., 1988;105(3-4):357-365..

16. Maeyama T. - A study of experimental sinusitis in rabbits. Auris Nasus Larynx, $1981 ; 8(2): 87-97$.

17. Gocea A., Taulescu M., Trombitas V., Albu S. - Effects of Cryotherapy on the Maxillary Antrostomy Patency in a Rabbit Model of Chronic Rhinosinusitis. Hindawi Publishing Corporation BioMed Research International, Volume 2013. Article ID 101534.

18. Stierna P., Kumlien J., Carlsoo B. - Experimental sinusitis in rabbits induced by aerobic and anaerobic bacteria: models for research in sinusitis. J Otolaryngol., 1991;20(6):376-378.

19. Westrin K.M., Norlander T., Stierna P., Carlsoo B., Nord C.E. Experimental maxillary sinusitis induced by Bacteroides fragilis: a bacteriological and histological study in rabbits. Acta Otolaryngol., 1992;112:107-114.

20. Cetin C.B., Kara C.O., Colakoglu N., Sengul M., Pinar H.S. - Experimental sinusitis in nasally catheterised rabbits. Rhinology. 2002;40(3):154-158.

21. Null G. - Ozone: A Wide-Spectrum Healer. www.scribd.com

22. Кудь А.А., Бархоткина Т.М. - Озонотерапия в ЛОР- практике. Озон в биологии и меднцине. Материалы 1-ой Украинско-Русской научнопрактической конференции. Одесса, 2003;р.102-104. 